\title{
Glycosylated Hemoglobin as a Surrogate for the Prevention of Cardiovascular Events in Cardiovascular Outcome Trials Comparing New Antidiabetic Drugs to Placebo
}

\author{
Pierre Ambrosi ${ }^{\mathrm{a}, \mathrm{b}}$ Aurélie Daumas ${ }^{\mathrm{a}}$ Patrick Villani ${ }^{\mathrm{a}}$ Roch Giorgi ${ }^{\mathrm{c}}$ \\ ${ }^{a}$ Department of Therapeutics, Marseille, France; ${ }^{b}$ Department of Cardiology, Hôpital de la Timone, Marseille,

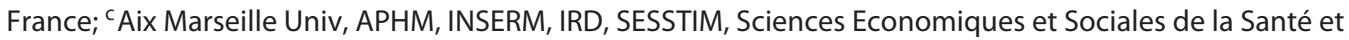 \\ Traitement de I'Information Médicale, Hop Timone, BioSTIC, Biostatistique et Technologies de I'Information et de la \\ Communication, Marseille, France
}

\section{Keywords}

Diabetes · Antidiabetic drugs · Placebo $\cdot$ Major adverse cardiac events $\cdot$ Glycosylated hemoglobin

\begin{abstract}
Background and Objectives: The value of glycosylated hemoglobin ( $\mathrm{HbA} 1 \mathrm{c})$ as a surrogate marker for the prevention of cardiovascular outcomes on antidiabetic drugs is debated. The 2008 FDA guidance led to multiple large clinical trials to evaluate the effect of new antidiabetic drugs versus placebo on major adverse cardiac events (MACE). The aim of this study was to evaluate the relation between MACE and $\mathrm{HbA} 1 \mathrm{c}$ decrease between antidiabetic drug and placebo across the spectrum of cardiovascular outcome trials (CVOT). Methods: In this systematic review, we included randomized controlled trials that compared an antidiabetic drug to placebo in addition to current standard of care with the primary intention of demonstrating cardiovascular safety. We investigated the relationship between MACE decrease on antidiabetic drug and $\mathrm{HbA} 1 \mathrm{c}$ reduction on antidiabetic drug using the coefficient correlation. We also studied the effects of potential confounders on MACE decrease. Results: Fourteen eligible trials including 128,149 patients were included, 12,114 of whom experienced MACE. Mean achieved HbA1c absolute reductions on antidiabetic treatment versus place-
\end{abstract}

bo varied from 0.29 to $1 \%$. The decrease of MACE on antidiabetic drug was significantly correlated with mean $\mathrm{HbA} 1 \mathrm{c}$ reduction $(r=0.88,95 \% \mathrm{Cl}: 0.67-0.96, p<0.001)$ and weight loss ( $r=0.81,95 \% \mathrm{Cl}: 0.46-0.94, p<0.001)$. In a bivariate model including weight loss, only $\mathrm{HbA} 1 \mathrm{c}$ reduction remained significantly correlated with the decrease of MACE on antidiabetic drug $(p=0.019)$. Conclusion: Across CVOT, the decrease in MACE incidence on various antidiabetic drugs is significantly correlated with $\mathrm{HbA} 1 \mathrm{c}$ reduction. This metaanalysis supports $\mathrm{HbA} 1 \mathrm{c}$ as an appropriate surrogate endpoint for cardiovascular events. Our analysis supports that changes in $\mathrm{HbA} 1 \mathrm{c}$ should be taken into account while interpreting effects of new antidiabetic drugs on cardiovascular outcomes.

(c) 2020 S. Karger AG, Basel

\section{Introduction}

Glycated hemoglobin (HbA1c) predicts the risk of microvascular and macrovascular outcomes in diabetes [1, 2]. Moreover, the DCCT study has shown that the decrease of $\mathrm{HbAlc}$ on intensive antidiabetic treatment is associated with lessening of complications in type 1 diabetes [3]. Other studies support the hypothesis of reduced microvascular complications with improved long-term

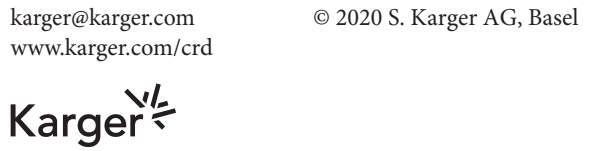


glycemic control in patients with type 2 diabetes [4]. For these reasons, $\mathrm{HbAlc}$ has been recognized by the Food and Drug Administration (FDA) and the European Medicines Agency (EMA) as a surrogate marker for preventing diabetes complications $[5,6]$.

However, accumulating data question the use of $\mathrm{HbAlc}$ as a surrogate [7]. In the ACCORD study, the use of intensive glucose-lowering therapy for 3.5 years increased mortality and did not significantly reduce major cardiovascular events as compared with standard therapy [8]. Moreover, in a meta-analysis by Nissen and Wolski [9], rosiglitazone treatment was associated with increased myocardial infarction despite a significant glucose-lowering effect.

In 2008, the FDA began to require trials to reasonably exclude an increased cardiovascular risk on new antidiabetic drugs submitted for approval [5]. This guidance led to multiple large clinical trials comparing the effect of these new antidiabetic drugs on major adverse cardiac events (MACE) versus placebo. In the present study, we evaluated the relation between MACE and HbAlc decrease across these trials, using trial-level data.

\section{Methods}

\section{Article Search and Selection}

We performed a systematic review following the Preferred Reporting Items for Systematic reviews and Meta-Analyses (PRISMA) guidelines for systematic review and meta-analyses [10]. A MEDLINE search identified trials in humans comparing an antidiabetic drug and placebo, published in full and in English between December 2008 (publication of FDA guidance) and July 2019. Eligible studies were of randomized, controlled design, reporting MACE, comparing an antidiabetic drug to placebo, in addition to current standard of care with the primary intention of demonstrating cardiovascular safety. The electronic search combined the keywords and MeSH terms: "alogliptin, anagliptin, gemigliptin, imagliptin, omarigliptin, saxagliptin, sitagliptin, teneligliptin, vildagliptin, canagliflozin, empagliflozin, dapagliflozin, ertugliflozin, ipragliflozin, luseogliflozin, tofogliflozin, sotagliflozin, albiglutide, dulaglutide, exenatide, liraglutide, lixisenatide, semaglutide, nateglinide, "antidiabetic," "glucose-lowering," "placebo," "cardiovascular," "randomized."

All citations were screened at the abstract level, and full articles of eligible trials were independently reviewed by two observers (P.A. and A.D.). These two authors extracted data from the included study in an unblinded fashion. From each eligible study, we determined the mean difference in MACE incidence and the mean difference in $\mathrm{HbA} 1 \mathrm{c}$ between the placebo and the antidiabetic arms during the treatment period. We also collected the following variables: number of patients per arm; length of treatment period; age; duration of diabetes; history of cardiovascular disease; mean $\mathrm{HbAlc}$ at baseline; number of patients reporting at least one major hypoglycemic event; between-group difference in weight variation; and all-cause mortality. The quality of individual trial design and execution was assessed via the modified Jadad scale [11]. Scores ranged from 0 to 8 with a higher score indicating higher quality.

\section{Endpoints}

Our prespecified primary analysis evaluated the association between mean $\mathrm{HbA} 1 \mathrm{c}$ reduction and relative variation in MACE incidence. MACE were defined as a composite cardiovascular death, nonfatal myocardial infarction, or nonfatal ischemic stroke. MACE numbers were extracted from the ITT analysis. Mean $\mathrm{HbA} 1 \mathrm{c}$ reduction was calculated in each trial as the difference in mean $\mathrm{HbA} 1 \mathrm{c}$ between placebo and antidiabetic groups during the whole follow-up as provided by the publication. When these data were not given, the mean difference in $\mathrm{HbAlc}$ was obtained from the HbAlc evolution graph at mid follow-up.

\section{Statistical Analysis}

The association between mean $\mathrm{HbAlc}$ reduction and relative variation in MACE incidence was quantified using the coefficient correlation, as their scatterplot indicated linear relation. We also tested the effect of factors (study duration, age, mean between arms difference in major hypoglycemia, weight loss, class of antidiabetic drug) possibly influencing the relative variation in MACE incidence. The association between mean $\mathrm{HbA} 1 \mathrm{c}$ reduction and relative variation in MACE incidence was finally studied in a bivariate regression analysis including the covariate (weight loss) significantly associated with relative variation in MACE incidence. The results were considered statistically significant with a $p$ value of $p \leq 0.05$. Statistical analysis was performed using the software program $\mathrm{R}$ (version 3.4.3).

\section{Results}

\section{Trial Characteristics}

We identified 14 randomized cardiovascular outcome trials (CVOT) which met the inclusion criteria and were included in the meta-analysis, yielding a total of 128,149 patients. The antidiabetic drugs were a dipeptidyl peptidase 4 (DPP-4) inhibitor: saxagliptin, alogliptin, sitagliptin, omarigliptin, linagliptin [12-16], an analogue of human glucagon-like peptide 1 (GLP-1): lixisenatide, liraglutide, semaglutide, exenatide, albiglutide [17-22] or an inhibitor of sodium-glucose cotransporter 2 (SGLT2): empagliflozin, canagliflozin, dapagliflozin [23-25]. In all these trials, other therapy for the management of the patient's diabetes was at the discretion of the responsible physician. Our search identified 1 trial still not published more than 2 years after completion of the study (FREEDOM-CVO) [26].

Table 1 lists the characteristics of the individual trials. In 5 trials, patients needed to have a history of cardiovascular disease to be included $[13,15,17,22,23]$. In the other 8 trials, eligible patients had either established cardiovascular disease or associated cardiovascular risk fac- 
Table 1. Characteristics of the 14 cardiovascular outcome trials

\begin{tabular}{|c|c|c|c|c|c|c|c|c|c|}
\hline Study & Drug & $\begin{array}{l}\text { Median } \\
\text { duration } \\
\text { (years) }\end{array}$ & $\begin{array}{l}\text { Age } \\
\text { (years } \pm \\
\text { SD) }\end{array}$ & $\begin{array}{l}\text { CVD } \\
(\%)\end{array}$ & $\begin{array}{l}\text { Diabetes } \\
\text { duration } \\
\text { (years) }\end{array}$ & $\begin{array}{l}\Delta \mathrm{HbA} 1 \mathrm{c} \\
(\%)\end{array}$ & $\begin{array}{l}\triangle \mathrm{MACE} \\
(\%)\end{array}$ & $\begin{array}{l}\Delta \text { hypoG } \\
(\%)\end{array}$ & $\begin{array}{l}\Delta \text { weight } \\
(\%)\end{array}$ \\
\hline EXAMINE (13) & alogliptin & 1.5 & 61 & 100 & 7.2 & 0.36 & 3 & 18 & 0 \\
\hline TECOS (14) & sitagliptin & 3.0 & $65 \pm 8$ & 74 & 11.6 & 0.29 & -1 & 12 & NA \\
\hline Gantz (15) & omarigliptin & 1.8 & $64 \pm 9$ & 100 & 12.0 & 0.3 & 0 & 26 & 0 \\
\hline ELIXA (17) & lixisenatide & 2.1 & $61 \pm 10$ & 100 & 9.3 & 0.3 & -2 & -41 & 0.8 \\
\hline LEADER (18) & liraglutide & 3.8 & $64 \pm 7$ & 82 & 12.8 & 0.4 & 12 & -25 & 2.5 \\
\hline SUSTAIN-6 (19) & semaglutide & 2.1 & $65 \pm 7$ & 72 & 14.0 & 1.0 & 26 & NA & 4 \\
\hline PIONEER 6 (20) & semaglutide & 1.3 & $66 \pm 7$ & 85 & 14.9 & 0.7 & 20 & 77 & 3.7 \\
\hline EXSCEL (21) & exenatide & 3.2 & 62 & 73 & 12.0 & 0.53 & 7 & 13 & 1.4 \\
\hline HARMONY (22) & albiglutide & 1.6 & $64 \pm 9$ & 100 & 14.1 & 0.58 & 21 & -43 & 0.8 \\
\hline
\end{tabular}

CVD, history of cardiovascular disease; $\triangle \mathrm{HbA} 1 \mathrm{c}, \mathrm{HbA1c}$ on placebo - HbA1c on antidiabetic drug; $\triangle \mathrm{MACE}$, relative decrease in MACE; $\triangle$ hypoG, mean relative variation in numbers of serious hypoglycemia; $\Delta$ weight, ([mean initial weight on antidiabetic - mean final weight on antidiabetic] - [mean initial weight on placebo - mean final weight on placebo])/mean initial weight on placebo. NA, not available.

tors. At inclusion, the mean age of the patients varied from 61 to 66 years and the mean diabetes duration varied from 7.1 to 14.7 years. The shortest trial mean treatment period was 1.3 year in PIONEER 6 and the longest was 5 years in DECLARE-TIMI 58. Baseline HbAlc ranged from 7.2 to $8.8 \%$. Among the 14 trials, the Jadad score was $\geq 7 / 8$, indicating a high methodological quality.

\section{Outcomes}

The mean absolute reduction in HbAlc on antidiabetic drug versus placebo in each trial varied from 0.29 to $0.36 \%$ on DPP- 4 inhibitor, 0.3 to $1 \%$ on GLP- 1 analogue, and 0.42 to $0.58 \%$ on SGLT-2 inhibitor (Table 1 ).

The variation of weight between the antidiabetic and placebo group was available from all trials except one [14]. The mean weight loss between the antidiabetic group and the placebo group varied from 0.0 to $3.6 \mathrm{~kg}$, corresponding to a mean relative weight loss of 0 to $4 \%$.

The numbers of patients with MACE were reported in all trials, and the total number of patients experiencing MACE was 12,114 . The incidence of MACE in the placebo group varied from 5.4 to $14 \%$. The incidence of MACE was significantly lower in the antidiabetic group than in the placebo group in 5 trials (LEADER, SUSTAIN-6, HARMONY, EMPA-REG, CANVAS). The relative decrease in MACE on antidiabetic drug versus placebo ranged from -3 to $26 \%$ (Table 1 ).

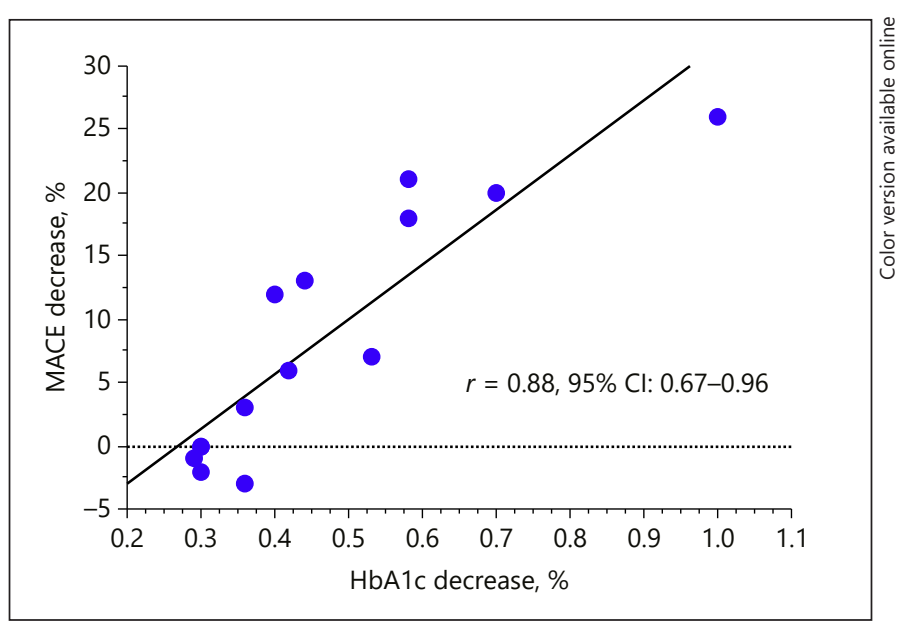

Fig. 1. Regression graph of MACE decrease versus $\mathrm{HbA1c}$ reduction on antidiabetic drug in the 14 CVOT.

\section{Relation between HbA1c Decrease and MACE}

According to the funnel plot, a publication bias seems unlikely (online suppl. material 1; for all online suppl. material, see www.karger.com/doi/10.1159/000506004). HbA1c reduction was significantly associated with MACE decrease $(r=0.88,95 \%$ CI: $0.67-0.96, p<0.001$; Fig. 1$)$ in univariate analysis. The covariates (age, percentage of patients with a history of cardiovascular disease, duration of diabetes, $\mathrm{HbAlc}$ at baseline, class of antidiabetic drug, study dura- 
tion, mean between arms difference in major hypoglycemia, relative weight loss, and class of antidiabetic drug) were obtained from all studies except for major hypoglycemia that was not reported in two studies $[19,24]$ and weight loss that was not reported in one study [14]. In univariate analysis, weight loss on antidiabetic drug versus placebo significantly correlated with relative variation in MACE incidence ( $r=0.81,95 \%$ CI: $0.46-0.94, p<0.001$; online suppl. material 2). In a bivariate model including weight loss, only $\mathrm{HbA1c}$ reduction remained significantly correlated with the decrease of MACE on antidiabetic drug $(p=0.019)$.

\section{Discussion}

The main result of this analysis including nearly 128,000 patients is that the decrease in MACE incidence is significantly correlated with $\mathrm{HbAlc}$ reduction on antidiabetic drug in CVOT. Moreover, this relation is still significant in a bivariate regression analysis including weight loss.

Some studies performed before 2008 have shown that an antidiabetic drug may have no effect on cardiovascular targets in spite of a significant $\mathrm{HbA1c}$ reduction. This observation has led to 2008 FDA guidance. The most striking evidence was the meta-analysis of Nissen and Wolski [9] showing that rosiglitazone treatment was associated with increased myocardial infarction incidence despite a significant glucose-lowering effect. However, the metaanalysis of Nissen and Wolski was not restricted to randomized trials. The RECORD study was specifically designed to test the cardiovascular safety of rosiglitazone [27]. This open-label trial did not find any significant difference in MACE between rosiglitazone and the control group ( $\mathrm{HR}=0.93,95 \% \mathrm{CI}: 0.74-1.15)$. One possible explanation is that the difference in $\mathrm{HbA} 1 \mathrm{c}$ between the two groups was too low $(0.3 \%$ mean absolute reduction on rosiglitazone versus control from baseline to 5-year follow-up). In the PROactive study, pioglitazone has been compared to placebo to investigate whether pioglitazone reduces macrovascular morbidity and mortality [28]. MACE were a secondary endpoint and were significantly decreased on pioglitazone (mean decrease $=16 \%, p=$ 0.027 ), while $\mathrm{HbA} 1 \mathrm{c}$ was significantly lower in the pioglitazone arm than in the placebo arm $(0.5 \%$ mean absolute reduction). This study was not included in our metaanalysis since it was published before the 2008 FDA guidance. Restricting our meta-analysis to CVOT published after this guidance allowed a high consistency in the methodology (drug compared to placebo, MACE as a primary endpoint) and a low publication bias.

MACE according to HbA1c Decrease
Most of the evidence supporting the usefulness of $\mathrm{HbAlc}$ as a surrogate for the reduction of cardiovascular events derived from the DCCT trial [3]. In the DCCT trial, 1,441 patients with type 1 diabetes were randomly assigned to intensive or conventional therapy, treating them for a mean of 6.5 years between 1983 and 1993. The main results of DCCT were that the mean glycosylated hemoglobin value was lower (7.4 vs. 9.1\%) in the intensive-treatment group, and significantly less patients had a cardiovascular event in the intensive-treatment group (31 vs. 52 patients). Our analysis finds an association between $\mathrm{HbAlc}$ decrease and MACE decrease in a much larger population not restricted to type 1 diabetes and in the modern therapeutic era. Moreover, our analysis shows the independency of this association when taking into account weight loss. Publication bias is unlikely since all CVOT of antidiabetic drugs have been registered and only one trial has still not been published more than 2 years after trial completion.

The main limitation of this study is the use of triallevel data rather than patient-level data which would allow a more robust analysis. Another limitation is the relatively low number of trials resulting in a lack of statistical power to evaluate the influence of various covariates such as antidiabetic class on MACE incidence.

\section{Conclusion}

Our analysis finds an association between $\mathrm{HbAlc}$ reduction and MACE decrease across CVOT and this association is still significant when taking into account weight loss. This result supports the usefulness of $\mathrm{HbAlc}$ as a surrogate marker for the prevention of cardiovascular outcomes. Our analysis supports that changes in $\mathrm{HbAlc}$ should be considered while interpreting the effects of new antidiabetic drugs on cardiovascular outcomes.

\section{Disclosure Statement}

The authors have no conflicts of interest to disclose.

\section{Author Contributions}

P.A. contributed to study design, data collection and interpretation, as well as writing and editing of the manuscript. A.D. contributed to study design and data collection. P.V. contributed to writing of the manuscript. R.G. contributed to the study design, data analysis, and data interpretation.

Cardiology 2020;145:370-374 


\section{References}

1 Stratton IM, Adler AI, Neil HA, Matthews DR, Manley SE, Cull CA, et al. Association of glycaemia with macrovascular and microvascular complications of type 2 diabetes (UKPDS 35): prospective observational study. BMJ. 2000 Aug;321(7258):405-12.

2 Selvin E, Marinopoulos S, Berkenblit G, Rami T, Brancati FL, Powe NR, et al. Meta-analysis: glycosylated hemoglobin and cardiovascular disease in diabetes mellitus. Ann Intern Med. 2004 Sep;141(6):421-31.

3 Nathan DM, Cleary PA, Backlund JY, Genuth SM, Lachin JM, Orchard TJ, et al.; Diabetes Control and Complications Trial/Epidemiology of Diabetes Interventions and Complications (DCCT/EDIC) Study Research Group. Intensive diabetes treatment and cardiovascular disease in patients with type 1 diabetes. N Engl J Med. 2005 Dec;353(25):2643-53.

4 Holman RR, Sourij H, Califf RM. Cardiovascular outcome trials of glucose-lowering drugs or strategies in type 2 diabetes. Lancet. 2014 Jun;383(9933):2008-17.

5 US Department of Health and Human Services, Food and Drug Administration, Center for Drug Evaluation and Research (CDER). Guidance for industry: diabetes mellitus evaluating cardiovascular risk in new antidiabetic therapies to treat type 2 diabetes. Available from: https://www.fda.gov/downloads/ Drugs/GuidanceComplianceRegulatoryInformation/Guidances/ucm071627.pdf.

6 European Medicines Agency. Guideline on clinical investigation of medicinal products in the treatment or prevention of diabetes mellitus. Available from: https://www.ema.europa.eu/docs/en_GB/document_library/Scientific_guideline/2012/06/WC500129256.pdf.

7 Lipska KJ, Krumholz HM. Is hemoglobin A1c the right outcome for studies of diabetes? JAMA. 2017 Mar;317(10):1017-8.

8 Gerstein HC, Miller ME, Byington RP, Goff DC Jr, Bigger JT, Buse JB, et al.; Action to Control Cardiovascular Risk in Diabetes Study Group. Effects of intensive glucose lowering in type 2 diabetes. N Engl J Med. 2008 Jun;358(24):2545-59.

9 Nissen SE, Wolski K. Effect of rosiglitazone on the risk of myocardial infarction and death from cardiovascular causes. N Engl J Med. 2007 Jun;356(24):2457-71.

10 Moher D, Liberati A, Tetzlaff J, Altman DG; PRISMA Group. Preferred reporting items for systematic reviews and meta-analyses: the PRISMA statement. Ann Intern Med. 2009 Aug;151(4):264-9.
11 Jadad AR, Moore RA, Carroll D, Jenkinson C, Reynolds DJ, Gavaghan DJ, et al. Assessing the quality of reports of randomized clinical trials: is blinding necessary? Control Clin Trials. $1996 \mathrm{Feb} ; 17(1): 1-12$.

12 Scirica BM, Bhatt DL, Braunwald E, Steg PG, Davidson J, Hirshberg B, et al.; SAVOR-TIMI 53 Steering Committee and Investigators. Saxagliptin and cardiovascular outcomes in patients with type 2 diabetes mellitus. N Engl J Med. 2013 Oct;369(14):1317-26.

13 White WB, Cannon CP, Heller SR, Nissen SE, Bergenstal RM, Bakris GL, et al.; EXAMINE Investigators. Alogliptin after acute coronary syndrome in patients with type 2 diabetes. $\mathrm{N}$ Engl J Med. 2013 Oct;369(14):1327-35.

14 Green JB, Bethel MA, Armstrong PW, Buse JB, Engel SS, Garg J, et al.; TECOS Study Group. Effect of sitagliptin on cardiovascular outcomes in type 2 diabetes. N Engl J Med. 2015 Jul;373(3):232-42.

15 Gantz I, Chen M, Suryawanshi S, Ntabadde C, Shah S, O'Neill EA, et al. A randomized, placebo-controlled study of the cardiovascular safety of the once-weekly DPP-4 inhibitor omarigliptin in patients with type 2 diabetes mellitus. Cardiovasc Diabetol. 2017 Sep; 16(1):112

16 Rosenstock J, Perkovic V, Johansen OE, Cooper ME, Kahn SE, Marx N, et al.; CARMELINA Investigators. Effect of linagliptin vs placebo on major cardiovascular events in adults with type 2 diabetes and high cardiovascular and renal risk. The CARMELINA randomized clinical trial. JAMA. 2019 Jan;321(1):6979.

17 Pfeffer MA, Claggett B, Diaz R, Dickstein K, Gerstein HC, Køber LV, et al.; ELIXA Investigators. Lixisenatide in patients with type 2 diabetes and acute coronary syndrome. N Engl J Med. 2015 Dec;373(23):2247-57.

18 Marso SP, Daniels GH, Brown-Frandsen K, Kristensen P, Mann JF, Nauck MA, et al.; LEADER Steering Committee; LEADER Trial Investigators. Liraglutide and cardiovascular outcomes in type 2 diabetes. N Engl J Med. 2016 Jul;375(4):311-22.

19 Marso SP, Bain SC, Consoli A, Eliaschewitz FG, Jódar E, Leiter LA, et al.; SUSTAIN-6 Investigators. Semaglutide and cardiovascular outcomes in patients with type 2 diabetes. $\mathrm{N}$ Engl J Med. 2016 Nov;375(19):1834-44.

20 Husain M, Birkenfeld AL, Donsmark M, Dungan K, Eliaschewitz FG, Franco DR, et al.; PIONEER 6 Investigators. Oral semaglutideand cardiovascular outcomes in patients with type 2 diabetes. N Engl J Med. 2019 Aug; 381(9):841-51.

21 Holman RR, Bethel MA, Mentz RJ, Thompson VP, Lokhnygina Y, Buse JB, et al.; EXSCEL Study Group. Effects of once-weekly exenatide on cardiovascular outcomes in type 2 diabetes. N Engl J Med. 2017 Sep;377(13): 1228-39.

22 Hernandez AF, Green JB, Janmohamed S, D'Agostino RB Sr, Granger CB, Jones NP, et al.; Harmony Outcomes committees and investigators. Albiglutide and cardiovascular outcomes in patients with type 2 diabetes and cardiovascular disease (Harmony Outcomes): a double-blind, randomised placebo-controlled trial. Lancet. 2018 Oct;392(10157): 1519-29.

23 Zinman B, Wanner C, Lachin JM, Fitchett D, Bluhmki E, Hantel S, et al.; EMPA-REG OUTCOME Investigators. Empagliflozin, cardiovascular outcomes, and mortality in type 2 diabetes. N Engl J Med. 2015 Nov; 373(22):2117-28.

24 Neal B, Perkovic V, Mahaffey KW, de Zeeuw D, Fulcher G, Erondu N, et al.; CANVAS Program Collaborative Group. Canagliflozin and cardiovascular and renal events in type 2 diabetes. N Engl J Med. 2017 Aug;377(7): 644-57.

25 Wiviott SD, Raz I, Bonaca MP, Mosenzon O, Kato ET, Cahn A, et al.; DECLARE-TIMI 58 Investigators. Dapagliflozin and Cardiovascular Outcomes in Type 2 Diabetes. N Engl J Med. 2019 Jan;380(4):347-57.

26 Intarcia Therapeutics. A study to evaluate cardiovascular outcomes in patients with type 2 diabetes treated with ITCA 650 . In: Clinical-Trials.gov (Internet). Available from: https://clinical trials.gov/show/ NCT01455896.

27 Home PD, Pocock SJ, Beck-Nielsen H, Curtis PS, Gomis R, Hanefeld M, et al.; RECORD Study Team. Rosiglitazone evaluated for cardiovascular outcomes in oral agent combination therapy for type 2 diabetes (RECORD): a multicentre, randomised, open-label trial. Lancet. 2009 Jun;373(9681):2125-35.

28 Dormandy JA, Charbonnel B, Eckland DJ, Erdmann E, Massi-Benedetti M, Moules IK, et al.; PROactive Investigators. Secondary prevention of macrovascular events in patients with type 2 diabetes in the PROactive Study (PROspective pioglitAzone Clinical Trial In macroVascular Events): a randomised controlled trial. Lancet. 2005 Oct; 366(9493):1279-89. 